\title{
Aplicação de sistemas dinâmicos na previsão de custos da produção
}

ViniciusA morim Sobreiro (USP) - sobreiro@sc.usp.br

Pedro Henriquede Sousa L eãoA raújo (USP) - pedro.leao@pepsico.com.br

M arcelo Seido Nagano (USP) - drnagano@usp.br

\section{Resumo}

O objetivo deste artigo foi aplicar a técnica de M odelagem de Sistemas D inâmicos - M SD para identificar os custos fixos, que na composição do custo total proporcionem viabilidade econômica à organização. A obtenção de tal informação possibilita uma empresa fixar preços, introduzir ou abandonar produtos como resposta aos rivais no mercado no qual está ou será inserida, além de ser uma perspectiva singular dos determinantes de desempenho das organizações. Para verificar tal aplicação, foi realizado um estudo de caso para a implementação de uma empresa envasadora de água mineral no Estado do Ceará. O s resultados evidenciaram que, mediante a MSD, é possível prever os custos da produção, auxiliando, assim, os gestores da organização quanto à viabilidade econômica.

Palavras-chave: Simulação, custos e modelagem de sistemas dinâmicos.

\section{APPLICATION OF DYNAMIC SYSTEMS TO PREDICT PRODUCTION COSTS}

\section{Abstract}

This paper aimed to apply the M odeling Dynamic Systems (M DS) technique to identify the fixed costs that, in total cost composition, would provide economic viability for an organization. This information enables an organization to fix prices and introduce or discontinue products as a response to competitors, besides providing a singular perspective of the performance determinants of such organization. Thus, a case study was carried out to set up a mineral water-producing organization in the state of Ceará. The results of this research showed that the MDS technique can satisfactorily predict production costs, thus guiding the organization's managers regarding economic viability.

Keywords: Simulation, costs and modeling of dynamic systems. 


\section{INTRO DUÇÃO}

$\mathrm{Na}$ atual conjuntura econômica, as organizações estão submetidas a um processo dinâmico de concorrência pelo mercado consumidor, e dentro desse contexto o sucesso ou fracasso é o resultado da habilidade em gerenciar, analisar e prever os custos do negócio para criar ou sustentar vantagens competitivas. Esse fato ocorre especialmente nas tomadas de decisões que envolvem o planejamento e implementação de unidades produtivas, pois se trata de projetos que, via de regra, envolvem considerado aporte de capital.

Os custos são compreendidos como medidas monetárias dos sacrifícios financeiros, ou seja, as informações referentes aos preços dos processos de transformações, que ocorrem nas organizações, nos governos, ou com as pessoas, a fim de atingir seus objetivos, por exemplo a obtenção de um produto ou serviço (BRUNI; FAMÁ, 2004). Além disso, Johnson e Kaplan (1991) demonstraram que esse conceito deve contemplar também a importância referente ao financeiro associado ao capital investido ou, de maneira mais apropriada, a remuneração do patrimônio líquido.

Nesse enfoque, os custos são classificados de forma generalizada quanto à variabilidade apresentada em relação às atividades das organizações, ou seja, as quantidades produzidas, comercializadas ou a prestação de serviço, em fixos e variáveis. Segundo Bruni e Famá (2004) e Bornia (2005), os custos fixos são aqueles que não variam e os custos variáveis, aqueles cujo valor se altera de forma direta em relação às atividades das organizações. Porém, Clark (1962) ressaltou que essa distribuição em classes dos custos deve também considerar o horizonte temporal, visto que determinados custos fixos podem ser considerados variáveis quando observados durante maiores períodos.

Um dos maiores problemas dos sistemas de custeio identificados pelos administradores, contadores e, sobretudo, por engenheiros mecânicos a partir do século XIX consiste na alocação dos custos fixos aos produtos (JOHNSON; KAPLAN, 1991; BRUNI; FAMÁ, 2004). Nesse contexto, os sistemas de custeio devem fornecer sinais relevantes sobre a posição competitiva das organizações dentro do mercado e não se limitarem à análise de eficiência das operações internas ou ao atendimento das exigências fiscais às quais as organizações possam ser submetidas.

Ainda nessa linha, Jones e Dugdale (2002) propuseram que sejam utilizados sistemas de custeios, ou seja, as maneiras pelas quais os custos são registrados, transferidos e atribuídos dentro da organização para solucionar tal problema. Nessa concepção, para Bruni e Famá (2004) tais técnicas apresentam diferenças apenas quanto ao rateio dos custos fixos, mais precisamente dos custos referentes à parte administrativa da organização. Embora o rateio dos custos fixos suscite inúmeras discussões, cabe ressaltar que Johnson e Kaplan (1991) afirmaram que, caso não exista grande heterogeneidade entre os produtos da organização, é possível aplicar práticas de custeio que relacionem os custos fixos aos produtos mediante volume, ordens de produção ou fabricação e processos.

Em uma perspectiva mais ampliada, conforme Drury e Tayles (2006), a pesquisa sobre custeios de produtos, mercadoria ou serviços apresentou tendências atuais para estudos nas seguintes áreas de pesquisa: (a) relação empírica sobre os dados da organização e os custos; (b) a utilização de níveis de informação; (c) aplicações em diferentes tipos de negócios; (d) identificação dos problemas na implementação de sistemas de custeio; e (e) identificação dos fatores de sucesso ou fracasso da implementação dos sistemas de custeio nas organizações. Contudo, nessas áreas, principalmente quanto à identificação dos fatores de sucesso de implementação dos sistemas de custeio, existem questões que ainda não foram abordadas, ou seja, lacunas que precisam ser preenchidas, a fim de contribuir para o desenvolvimento das práticas contábeis. Nessa concepção, o objetivo deste artigo foi responder ao seguinte problema de pesquisa: É possível prever os valores, dentro de um intervalo, dos custos fixos que, acrescidos aos custos variáveis, proporcionem a viabilidade econômica a um empreendimento mediante as técnicas de MSD, ou seja, integrar a análise de custos com análise dinâmica?

Para tanto, é realizado um estudo de caso para implementação de uma unidade envasadora de água mineral no Estado do Ceará, por meio da utilização do software STELLA, considerando-se as seguintes variáveis: (a) preço de venda; (b) demanda; e (c) custos de insumos de produção, bem como os critérios quanto às escolhas das fontes e às formas de tratamento das informações. Cabe ressaltar que a escolha da implementação de uma empresa de água mineral no Ceará está centrada na possibilidade de esse Estado se tornar um dos maiores consumidores de água mineral no mercado brasileiro.

Os resultados obtidos pela aplicação da técnica de MSD no custeio do envasamento de água mineral auxiliam, de maneira notável, os gestores responsáveis pelo empreendimento a tomarem as melhores decisões possíveis com relação a todos os riscos e incertezas. Além disso, é relevante salientar a importância desta pesquisa, visto que estuda o emprego de capital na Região Nordeste. 
O artigo está estruturado da seguinte forma; na próxima seção são apresentados os conceitos de MSD e a importância da identificação dos custos para os sistemas de produção, visando à tomada de decisão. Na seção 3 são expostos as condições e critérios para aplicação da técnica de MSD na previsão dos custos. Na seção 4 é apresentado o estudo de caso. Em seguida, na seção 5 se demonstram os resultados e finalmente, na seção 6 , as discussões e considerações finais.

\section{REVISÃO DA LITERATURA}

Para compreender a integração da análise de custos com as técnicas relacionadas à dinâmica de sistemas, é apresentado um exemplo bem detalhado dessa junção. Para tanto, há necessidade da exposição dos fundamentos normativos que estruturam os raciocínios relevantes a esses dois campos da ciência nesta seção.

\subsection{MSD}

A MSD surgiu com Forrester (1961), ou mais especificamente mediante estudos realizados nas tomadas de decisões sobre o inventário e recrutamento na General Eletric. Conforme Dyson e Chang (2005), essa modelagem oferece a melhor compreensão sobre os problemas complexos e, além disso, segundo Meadows et al. (1972), os modelos mentais elaborados pelo ser humano são inferiores quando comparados com os modelos computacionais, devido à dificuldade apresentada na compreensão do comportamento dos processos de realimentação ao longo do tempo.

Nesse sentido, Dyson e Chang (2005) propuseram a construção de diagramas de loops ou fluxo e estoque, a fim de facilitar a superação das limitações humanas no entendimento dos conceitos de MSD. Na construção desses diagramas é necessária a compreensão dos quatro componentes envolvidos na modelagem:

I Os estoques: abrangem as variáveis armazenadas de forma potencial para a utilização por outros componentes da modelagem.

I Os fluxos: representam as variáveis responsáveis pela modificação dos estoques.

I Os auxiliares: têm como função definir as equações algébricas dos fluxos, estoque ou outros auxiliares, mediante operações algébricas.
I Os auxiliares: têm como função definir as equações algébricas dos fluxos, estoque ou outros auxiliares, mediante operações algébricas.

I Os conectores: possuem a função de inter-relacionar todos os componentes do sistema, com o propósito de formar as equações algébricas.

Considerando tais definições, a Figura 1 ilustra os quatro componentes necessários à construção do modelo consoantes ao proposto por Dyson e Chang (2005).

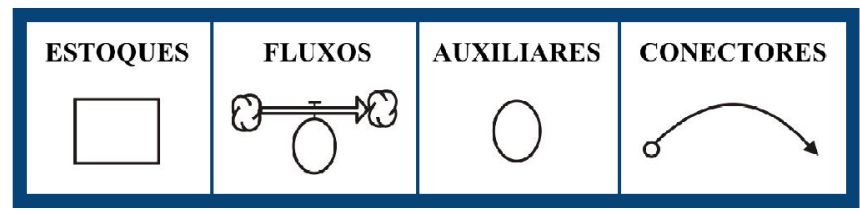

Figura 1 - Componentes para representação gráfica.

Tomando como base uma perspectiva cronológica, inicialmente se tem notação de MSD aplicada à simulação e previsão de sistemas socioeconômicos (FORRESTER, 1969, 1971; MEADOWS, 1973). Posteriormente, surgiram diversas pesquisas aplicando a modelagem em sistemas ambientais e avaliações de eficiências (VIZAYAKUMAR; MOHAPATRA, 1991, 1993; VEZJAK et al., 1998; FORD, 1999; WOOD; SHELLEY, 1999; ABBOTT; STANLEY, 1999; DEATON; WINEBRAKE, 2001; GUO et al., 2001). Ademais, Nail et al. (1992) se beneficiaram da modelagem para auxiliar o processo de tomadas de decisão no âmbito político, enquanto Grant et al. (1997) voltaram a relacionar MSD com o meio ambiente, mediante aplicações em sistemas ecológicos.

A agricultura, no entanto, também foi campo de pesquisa para as aplicações de Qu e Barney (1998) e Saysel et al. (2002), que simularam sistemas agrícolas quanto aos aspectos intrínsecos, como a safra; e extrínsecos, como o clima. Ainda, em uma vertente mais empresarial, Souza e Carpinetti (2005) utilizaram a MSD na análise de reprojeto para empresas, visto que estas necessitam de abordagens capazes de incorporar pontos de vista holísticos, incluindo considerações sociais, orgânicas e também psicológicas, além do foco técnico econômico oferecido por meio das técnicas tradicionais.

Recentemente, a MSD chegou ao mundo dos negócios auxiliando o processo de tomada das decisões em ambiente corporativo e à gestão de manufatura, visando estratégias eficientes de mercados (STERMAN, 2000; ADAMIDES; VOUTSINA, 2006).

Para a construção do modelo proposto por Dyson e Chang (2005), é necessário seguir algumas regras, de acordo com Flood e Jackson (1991), a fim de obter a 
maior exatidão possível da representação ou interpretação simplificada da realidade:

I Os estoques somente poderão ser precedidos por fluxos e sucedidos pelos auxiliares ou fluxo.

I O auxiliar poderá ser seguido por outro auxiliar ou um flux.

I O fluxo poderá ser seqüenciado por um estoqu.

I Um estoque não pode ser diretamente afetado por outro estoque.

Ao estruturar tais componentes, com o objetivo de realizar a simulação, obtém-se um diagrama de estoque e fluxo, conforme apresentado na Figura 2.

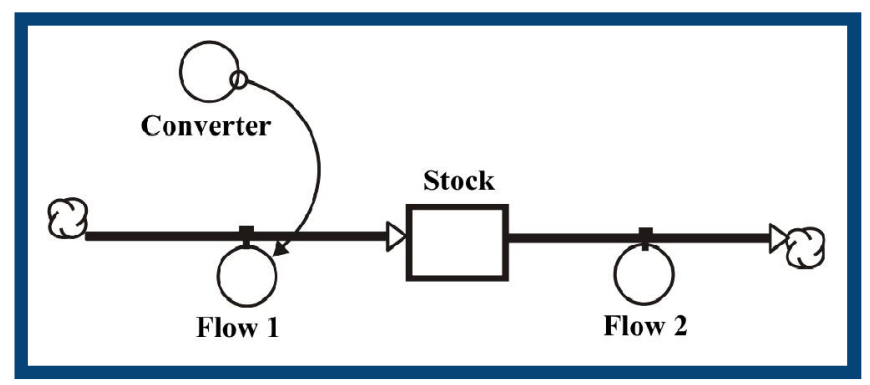

Figura 2 - Diagrama de estoque e fluxo.

Fonte: DYSON; CHANG, 2005.

O desenvolvimento de um diagrama conforme a Figura 2 possibilita que o gestor simule o objeto de estudo $\mathrm{e}$, assim, interprete ou identifique a forma mais segura às incertezas, pois, segundo Reibstein e Day (1999) e Rizzo et al. (2006), o diagrama permite:

I Compreender o efeito do tempo nas variáveis, mediante o fato de que o comportamento de vários anos possa ser reproduzido em poucos minutos.

I Reduzir os custos, pois a simulação acontece em ambiente computacional sem a necessidade de constituição física do objeto de estudo.

I Promove à criatividade: por meio da inexistência de risco, o usuário pode verificar o comportamento das variáveis quando submetidas às novas condições.

I A realização de vários ensaios, possibilitando aos gestores uma compreensão clara da natureza íntima do objeto estudado.

I Unificar opiniões divergentes entre os gestores, pois o problema é apresentado de forma única.

I Capturar e aplicar o conhecimento em apenas um repositório de informações competitivas.

Com o intuito de ressaltar a vantagem da utilização de MSD, discute-se na seção posterior como a modelagem pode ser utilizada para definir um de intervalos referentes aos custos fixos, objetivando gerar informa- ções de caráter estratégico para auxiliar os gestores na tomada de decisões.

\subsection{Custo e MSD}

$\mathrm{O}$ fato de que o custeio dos produtos contribui ou apóia a tomada de decisão nas funções de gestão e controle na administração das empresas é um acontecimento recente, visto que remonta, segundo Johnson e Kaplan (1991), às últimas décadas do século XIX. Entretanto, mesmo que se saiba que os primeiros registros de custos conhecidos pelos historiadores americanos, o da Boston Manufacturing Company, em Waltham no Massachusetts, apenas serviam para indicar a rentabilidade global da empresa mediante a avaliação dos estoques.

$O$ custeio da produção era, de início, uma ferramenta para facilitar a coordenação de diferentes atividades. Entretanto, devido ao surgimento de linhas de produção que contemplavam muitos produtos, diferenciando-se da produção fordista, esse aparecimento acabou se tornando um princípio condutor para monitorar a eficiência de todas as atividades realizadas, bem como e vincular o desempenho de cada processo à rentabilidade global da organização.

Ao se analisar as mudanças dos últimos anos em âmbito de desenvolvimento dos processos produtivos, a dedução mais evidente, segundo Spedding e Sun (1999), está centrada no fato de as organizações necessitarem de constantes atualizações; visto que a única coisa imutável no ambiente de concorrência são as mudanças nos níveis da competitividade. Esse fato justifica o ponto de vista apresentado por esses mesmos autores em trabalho a respeito de simulação dos eventos discretos para a utilização do custeio baseado em atividade em sistemas de produção. Fundamenta-se, assim, a possível utilização de MSD na identificação dos custos, visto que essa conciliação contemplará aspectos dos sistemas de produção e financeiros.

É importante ressaltar que os sistemas de custo devem fornecer sinais relevantes da posição competitiva das organizações dentro do mercado e não se limitarem à análise de eficiência das operações internas. Além do que a eficiência obtida por intermédio da precisa definição dos parâmetros operacionais do processo produtivo é privada caso a mensuração dos custos seja realizada de forma errada, ou sem a eleição das variáveis mais representativas (DHAVALE, 1992). Conseqüentemente, ainda nessa linha, importantes implicações gerenciais ficam omitidas ou distorcidas atrás de falhas na projeção 
das peculiaridades do sistema produtivo. Entre os principais sistemas desenvolvidos e apresentados pela literatura sobre custeios desde 1925, destacam-se:

। Custeio por departamentos: esse sistema relaciona o controle dos custos incorridos aos departamentos da organização ou aos centros de custos das organizações (NEVES; VICECONTI, 2003).

I Custeio por processos: neste sistema, os custos são determinados às categorias por tipo de gasto (natureza contábil). Além disso, são compilados por processos específicos e, posteriormente, distribuídos às unidades produzidas (BRUNI; FAMÁ, 2004).

I Custeio por ordens de produção ou fabricação: quando a organização apresenta um processo produtivo heterogêneo, o sistema de custeio é estruturado por meio das ordens referentes à produção para estoque ou encomenda expedida (JOHNSON; KAPLAN, 1991).

I Custeio-padrão: são os custos estabelecidos pela organização como metas para os produtos da linha de fabricação, levando-se em consideração as características tecnológicas do processo produtivo de cada um, a quantidade e preços dos insumos para a produção e os respectivos volumes desta. Tal técnica apresenta aplicação na aferição de desempenho, elaboração de orçamentos e orientação para formação de preços (NEVES; VICECONTI, 2003).

I Custeio variável: neste sistema, apropriam-se aos produtos, mercadorias e serviços vendidos apenas os custos variáveis, ou seja, apenas aqueles que estão diretamente relacionados com o volume de produção ou vendas (SANTOS, 2005).

I Custeio baseado em volume: nesta técnica, a soma dos custos fixos é rateada pelo volume de produção aos produtos da organização (BRIERLY et al., 2006).

I Custeio baseado em atividade: neste sistema, os custos são reportados aos produtos segundo o número de atividades responsáveis pelo consumo de recursos produtivos (TSAI; LAI, 2006).

Considerando que, segundo Johnson e Kaplan (1991) e Lucas (2002), todas as informações de custos e mix de produção consistem nos principais fomentos para as decisões de gerenciamento contábil Drury e Tayles (2006), em recente survey realizado com 184 organizações do Reino Unido, constataram que apenas $35 \%$ das empresas consultadas consideram outros custos além dos variáveis. Ainda, nesse enfoque, outro ponto importante consiste na comparação das práticas de custeios dos produtos em diferentes processos de fabricação, pois, conforme constatado por Brierley et al. (2006), em survey realizada com 280 membros da Chartered Institute of
M anagement Accountais (CIMA), não existem diferenças consideráveis entre as práticas de fabricação vigentes nas indústrias manufatureiras.

Nesse sentido, a importância desta pesquisa se fundamenta na exposição de processos de simulação de custos pelas organizações por meio de MSD. Em razão desse motivo, a seção seguinte expõem os métodos, ou seja, os critérios e condições para o estudo de caso.

\section{MÉTODOS}

\subsection{Variáveis e Q uestionário de Coleta de Dados}

De certa maneira, a coleta de dados foi realizada mediante o desenvolvimento das seguintes atividades:

I Seleção do instrumento ou método de coleta de dados: nesse sentido foi empregado um questionário desenvolvido pelos próprios alunos. O questionário era composto de questões abertas e fechadas, visando, assim, a obtenção de informações de teor quantitativas e qualitativas. Cabe aqui salientar que não houve a definição de uma amostra, pois se primou pela realização do maior número de entrevista dentro do período de 23 dias e nem a realização de um questionário-teste, mas sim a supervisão dos docentes ligados ao Departamento de Engenharia de Produção de universidade no Estado do Ceará.

I Aplicação do instrumento de coleta de dados: a aplicação deste instrumento ocorreu mediante entrevista agendada, anteriormente, por telefone e e-mail, com funcionários de organizações semelhantes e do sindicato.

I Preparação das observações: todos os questionários foram tabulados em planilhas eletrônicas e, conseqüentemente, comparados, visando à identificação de erros e dados digitados incorretamente.

Considerando os apontamentos anteriores, os principais dados e variáveis coletadas pelo grupo de estudante, mediante principalmente os questionários, foram: I Dados quanto às possíveis quantidades demandadas

I Informações referentes aos preços de comercialização praticados no mercado.

I Principais componentes dos custos variáveis relacionados aos produtos, a exemplo do custo das embalagens plásticas, tampas plásticas, rótulos, filme de polietileno, custo da tinta para impressão da validade e mão-de-obra direta. 
I Instrução quanto aos seguintes componente dos custos fixos: depreciação das máquinas, configuração das máquinas, amortização dos sistemas operacionais, aluguel do prédio, valores referentes a seguros e segurança, valores referentes à mão-de-obra indireta, logística interna e externa, codificação de produtos, atualização de dados e sobre ferramentas e outros dispositivos necessários para a gestão da organização.

É válido ressaltar que, no tocante aos alunos de graduação, por intermédio de uma empresa júnior instalada no campus da universidade, esses docentes, em fase final do curso de graduação, prestam consultorias a pequenos e médios investidores que, têm interesse em instalar unidades produtivas diversas (bens e, ou, serviços), ou, ainda, melhorar seus processos já em operação. Além disso, convém destacar que o estado do Ceará foi escolhido para implementação de mais uma unidade envasadora de água mineral por causa, principalmente, do mercado consumidor.

\subsection{Estudo de caso}

A partir da compreensão dos fundamentos que estruturam raciocínios relevantes, a MSD, os de custos, e da necessidade de integração desses dois campos do conhecimento, justifica-se a condução desta pesquisa, cujos procedimentos metodológicos são classificados com o estudo de caso, visto que o objeto desta pesquisa é observado de forma única e intensa.

O detalhamento sobre os procedimentos, ou seja, os critérios e condições utilizados e o fornecimento de justificativas sobre eles são apresentados no Quadro 1, com o propósito de alcançar o objetivo deste artigo.

O procedimento adotado para analisar os dados obtidos com a simulação dos custos fixos consiste na verificação do lucro, por meio da provável receita e custos totais, considerando-se todas as demais informações obtidas pelo grupo de estudantes.

A análise a seguir tomou como ponto de partida o interesse de um investidor em instalar uma unidade envasadora de água mineral. Exercendo o papel de investidor, o interessado questionou a viabilidade da implementação de uma fábrica para produzir tal produto.

Segundo Rosa et al. (2006), o último relatório anual, elaborado pelo Banco Nacional de Desenvolvimento Econômico e Social-BNDES, que apresenta um panorama do setor de bebidas no país, indicou que a maior parcela dos custos das águas envasadas corresponde aos insumos e serviços adicionados. Nessa perspectiva, a análise realizada tomou como base os pertinentes custos fixos e custos variáveis de produção. Sabe-se que existem diversos outros custos e despesas que inferem no processo de fabricação e composição do preço. Porém, este estudo limitou-se a considerar custos fixos e variáveis.

Quadro 1 - Detalhamento dos procedimentos

\begin{tabular}{|c|c|c|c|}
\hline \multirow{5}{*}{ 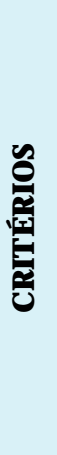 } & ETAPAS & PROCEDIMENTO & JUSTIFICATIVA \\
\hline & 1 & $\begin{array}{l}\text { Eleição dos parâmetros, } \\
\text { ou seja, os dados a serem } \\
\text { considerados }\end{array}$ & $\begin{array}{l}\text { Seleção das informações, ou de uma maneira mais apropriada, dos dados que serão } \\
\text { coletados pelos alunos, ou seja, das variáveis demanda, receita, custo de produção e } \\
\text { custos fixos }\end{array}$ \\
\hline & 2 & Coleta dos dados. & $\begin{array}{l}\text { Os dados foram coletados pelos alunos em fontes governamentais de acesso livre pela } \\
\text { internet e dos concorrentes, mediante pesquisa de campo, durante o período de } 23 \text { dias. }\end{array}$ \\
\hline & 3 & $\begin{array}{l}\text { H ardwares, softwares e } \\
\text { laboratório }\end{array}$ & $\begin{array}{l}\text { Escolha dos equipamentos de hardwares, softwares para realizar a simulação e o } \\
\text { espaço físico, ou seja, o laboratório para realização da simulação. }\end{array}$ \\
\hline & 4 & $\begin{array}{l}\text { Delimitação da } \\
\text { capacidade produtiva do } \\
\text { empreendimento }\end{array}$ & Delimitação e aquisição das máquinas apropriadas para o processo produtivo \\
\hline \multirow{3}{*}{ 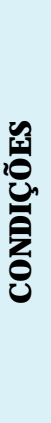 } & 5 & Padronização dos dados & $\begin{array}{l}\text { - } \quad \text { Foram escalonados } \\
\text { - } \quad \text { Agrupados de forma mensal } \\
\text { - Sofreram alterações quanto ao formato para servirem como inputs ao software, ou } \\
\text { seja, as vírgulas foram substituídas por pontos }\end{array}$ \\
\hline & 6 & Validação dos dados & $\begin{array}{l}\text { - Os dados foram validados segundo informações obtidas dos concorrentes e } \\
\text { mediante orientação dos professores do Departamento de Engenharia de Produção }\end{array}$ \\
\hline & 7 & $\begin{array}{l}\text { Hardwares, softwares e } \\
\text { laboratório }\end{array}$ & $\begin{array}{l}\text { - Hardware: AMD Duron 1,10 GHz, } 376 \mathrm{MB} \text { de Ram } \\
\text { - Software: Sistema operacional: Microsoft Windows XP Profissional versão } 2002 \\
\text { - } \quad \text { Instalações físicas: Laboratório do Departamento de Engenharia de Produção }\end{array}$ \\
\hline
\end{tabular}


Primeiramente, foi realizado um estudo de mercado com o objetivo de adquirir informações com bases nas fábricas concorrentes do mesmo ramo de negócio água mineral envasada. Este estudo de mercado foi consolidado por intermédio de pesquisas de campo, visitas ao sindicato regente do setor e de consultas em pesquisas de caráter oficial, elaboradas sob encomenda por parte daqueles que pretendem conhecer com mais afinco o ramo de negócio que se pretende atuar. Cabe ressaltar que não foram realizados gastos com aquisição de informação alguma. Assim, todas as informações utilizadas para a análise foram obtidas de maneira gratuita e legal. Também, é válido salientar que o objetivo de uma empresa júnior encubada em uma universidade não é a obtenção de superávits econômicos, mas sim a geração e propagação de todas as formas de conhecimento.

A pesquisa de mercado realizada identificou que a demanda no Brasil por águas envasadas apresentou constante crescimento nos últimos anos. Segundo estatísticas do Departamento Nacional de Produção Mineral (DNPM) e da Associação Brasileira da Indústria de Águas Minerais (ABINAM), o volume da produção de água envasada cresceu a uma média anual de $15 \%$, de 1990 até 2006. De acordo com os levantamentos da associação, a produção de água envasada, em 2004, foi de 5,1 bilhões de litros. Quanto ao faturamento em 2004, a indústria brasileira de águas envasada apresentou $\mathrm{R} \$ 1,2$ bilhão.

A pesquisa de mercado serviu para definir o setor de bebidas, mais especificamente o ramo de águas minerais. Entre todas as informações coletadas, as mais relevantes foram:

I Produção nacional (em 2004): 5,1 bilhões de litro.

I Região líder em produção: Sudeste.

I Estado líder em produção: São Paulo.

I Posicionamento da Região Nordeste no ranking brasileiro de produção: $2^{\circ}$.

I Prospecção de aumento de produção para 2006/2007: $12 \%$.

I Marketshare: 13 empresas detêm 35\% do mercado. Os 65\% restantes são detidos por mais de 200 engarrafadoras, muitas delas com atuação local.

I Principais empresas: Grupo Edson Queiroz (Indaiá e Minalba), Flamin Mineração (Lindoya Bioleve), Empresa de Águas Ouro Fino (Ouro Fino), e Grupo Schincariol (Schincariol).

I Grupo líder: Edson Queiroz (40\% das regiões Nordeste e Centro Oeste).

I Formas de embalagens: garrafão de 20 L (50,2\%), garrafas PET $(34,2 \%)$, vidro $(10,4 \%)$, copos plásticos $(3,4 \%)$ e outros $(1,8 \%)$.
I Nicho de maior crescimento: garrafão de 20 L.

I Produção Nordeste: 18,5\%.

1 Custo médio de produção: custo variável de $500 \mathrm{~mL}$ sem gás: $\mathrm{R} \$ 0,33$.

Dispostos dessas informações, este estudo foi definido como uma simulação para calcular um intervalo no qual o montante atribuído ao custo fixo que, acrescido dos custos variáveis de produção, pode se alterar sem inviabilizar o preço de venda do produto. Quanto aos custos diretos, foram considerados como insumos: (a) a préforma de polietileno tereftalato - PET de gramatura 16,5 g; (b) tampa de plástico de polipropileno; (c) rótulo de polipropileno bi-orientado - BOPP; (d) filme de polietileno; e (e) mão-de-obra.

Sabe-se que existem mais insumos que compõem o produto como a tinta desk jet para a impressão do prazo de validade e do lote de produção, a água, entre outros. Porém, de acordo com a participação de cada insumo no produto final, os dados de custeio fixo considerados compõem $93 \%$ do custo direto total da confecção do produto. Não obstante, durante a pesquisa se constatou que a água não compõe o custo do produto na maioria das empresas do ramo, devido ao fato de provir de poços naturais. Assim, seu custo como insumo não é contabilizado, tratando-se de uma medida estratégica para se obter um preço de produto mais competitivo.

$\mathrm{Na}$ esteira desse processo foi definido o construto relacional, conforme apresentado na Figura 3, com o objetivo de alinhar, de maneira estratégica, as técnicas de MSD com o estudo de caso sob questão, estudo esse dividido em três grandes fases, a fim de estabelecer prazos para realização ou o caminho crítico de todos os processos.

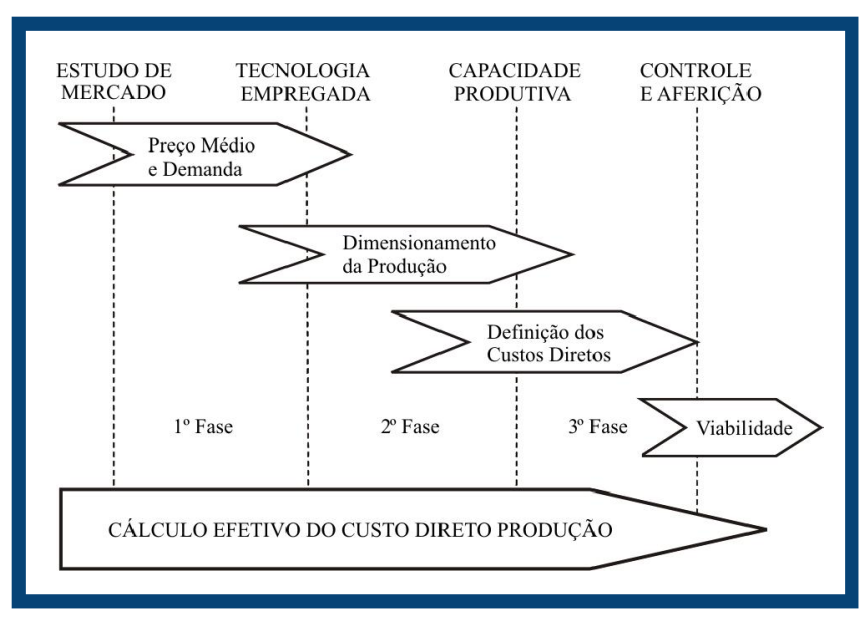

Figura 3 - Fases para cálculo dos custos no processo de produção. 
Nesse contexto, segundo Nosbisch e Winter, (2006), a primeira fase consiste na pesquisa de mercado para a obtenção do preço médio para o produto. Não obstante os preços dos concorrentes o preço médio obtido foi de $\mathrm{R} \$ 1,15$. Todavia, ainda nessa fase foi realizada a pesquisa para identificar uma provável demanda para o produto; a quantidade prevista está dentro do intervalo de 50.000 a 90.000 unidades.

Posteriormente, a segunda fase consistiu no dimensionamento da produção, ou seja, na aquisição de máquinas e equipamento necessários para atender a essa capacidade. Assim, foi estabelecido, inicialmente, uma capacidade para atender a uma demanda de 40.000 unidades, entretanto a capacidade máxima de produção está em 60.000 unidades.

Finalmente, na terceira fase todos os principais custos diretos de produção foram obtidos, conforme demonstrado na Tabela 1.
Com base nas informações apresentadas na Tabela 1, foi possível, por meio de simulação, calcular o intervalo de custo fixo que, quando acrescido dos custos variáveis, ou seja, $\mathrm{CT}=\mathrm{CV}+\mathrm{CF} \Rightarrow \mathrm{CT}=0,2451+\mathrm{CF}$, possibilita viabilidade econômica para implementação considerando-se a capacidade produtiva de 60.000 unidades, com preço médio de mercado de R $\$ 1,15$ para o produto. A representação da simulação por meio de MSD, considerando-se o construto relacional exposto na Figura 3, encontra-se na Figura 4.

Cabe ressaltar que todas as fases demonstradas na Figura 3 são identificadas na Figura 4. Tal fato se estabelece porque o software Stella 5.0 possibilita ao usuário construir um ambiente que relacione o construto relacional com a modelagem dinâmica. Além disso, o software não necessita da inserção pelo usuário das equações de dinâmica quanto ao objeto de estudo, pois, ao fazer o relacionamento dos componentes de representação gráfica, o próprio software faz a construção, ou melhor, a elaboração dessas equações.

Tabela 1 - Custos diretos de produção

\begin{tabular}{|l|c|}
\hline \multicolumn{1}{|c|}{ INSUM OS } & CUSTOS \\
\hline Filme de polietileno & 0,0034 \\
\hline Pré-forma & 0,1460 \\
\hline Rótulo & 0,0430 \\
\hline Tampa & 0,0510 \\
\hline Mão de obra & 0,0017 \\
\hline TOTAL UNITÁRIO & 0,2451 \\
\hline
\end{tabular}

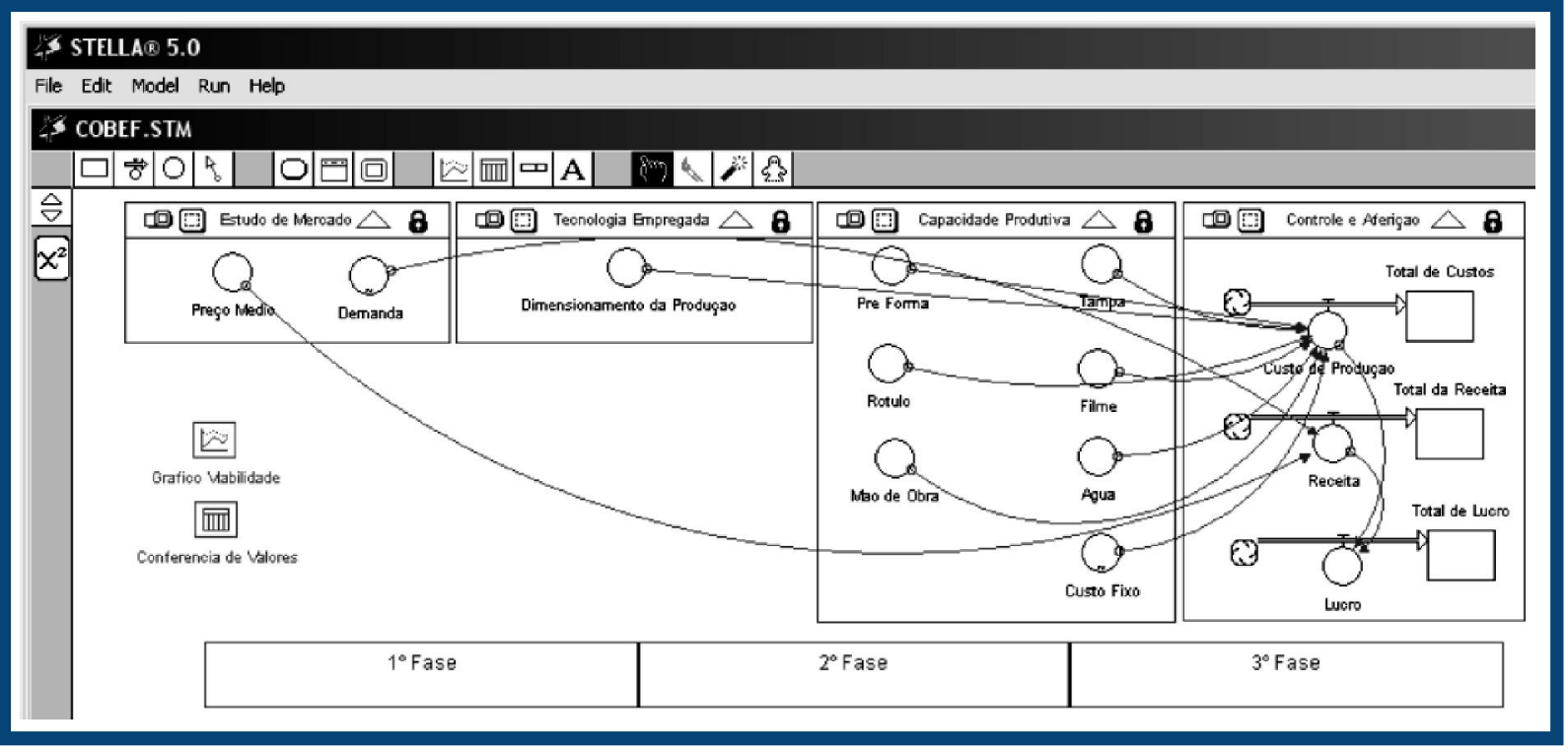

Figura 4 - Simulação por meio de técnicas de MSD. 
Vinicius Amorim Sobreiro

Pedro Henrique de Sousa Leão Araújo

Marcelo Seido Nagano

\section{RESULTADOS}

Com o objetivo de prever os custos fixos utilizando MSD que favoreçam o empreendimento quanto à viabilidade econômica considerando os métodos expostos no Quadro 1 os resultados obtidos por meio da simulação são apresentados na Figura 5, que representa uma tabela elaborada pelo software utilizado. Nessa figura, é possí- vel observar o comportamento das variáveis durante os processos de interação e constatar que a implementação dessa organização para o envasamento de água mineral será apenas viável, caso os custos fixos estejam no intervalo de $\mathrm{R} \$ 40.000,00$ a $\mathrm{R} \$ 51.000,00$, condizente com a demanda e capacidade produtiva estabelecidas.

Os resultados obtidos evidenciam o comportamento da realidade ou o modo de referência das variáveis e suas interações. Tal situação é demonstrada na Figura 6.

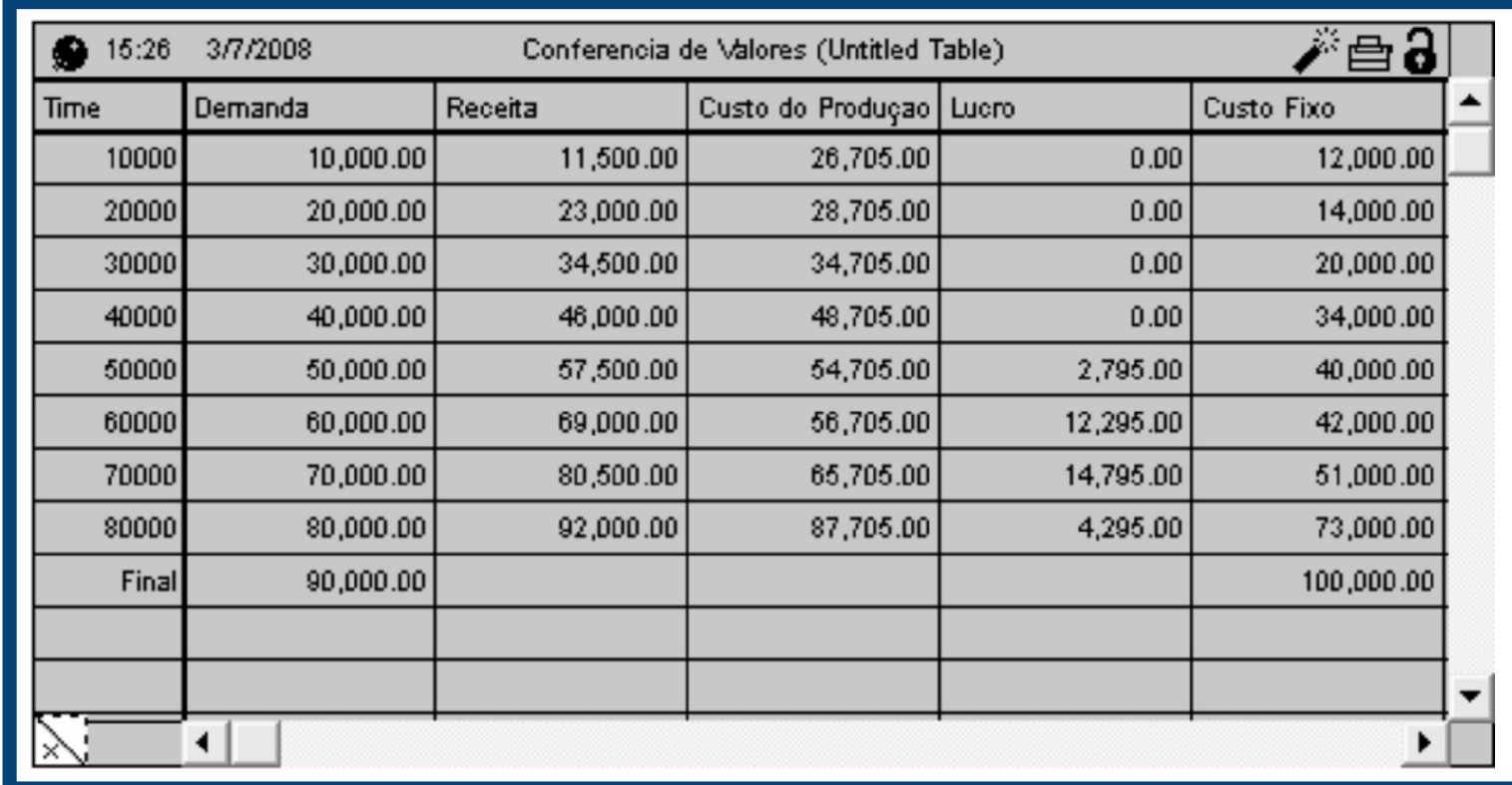

Figura 5 - Tabela de resultados.

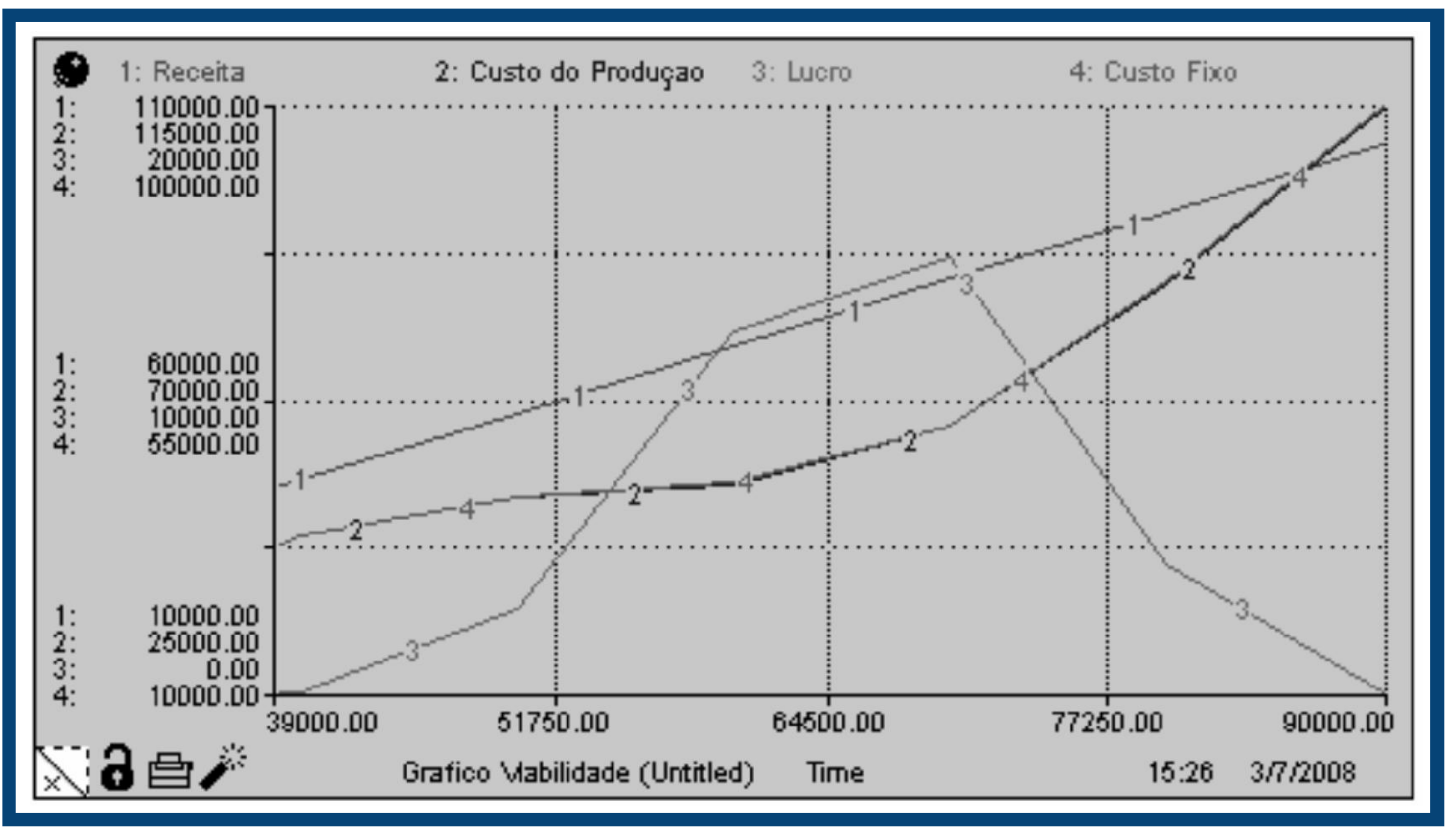

Figura 6 - Gráfico dos resultados obtidos. 
Com base, no entanto, nos valores obtidos, o custo do produto é de aproximadamente $\mathrm{R} \$ 0,9118$, o que, quando comparado com o preço médio de venda de $\mathrm{R} \$ 1,15$, proporcionou uma margem de lucro aproximada de $20,71 \%$, sem considerar os impostos, contribuições ou demais remunerações ao patrimônio líquido.

Ainda considerando as informações anteriores, o software Stella 5.0 apresenta todas as informações quanto às variáveis modeladas e, conseqüentemente, as equações resultantes de suas iterações, conforme apresentado na Figura 7.

Apenas para efeitos de demonstração da facilida- de e da possibilidade de simulação quanto a alterações das principais variáveis, na Figura 8 apresentam-se os dados considerando, agora, dois níveis de custos fixos, ou seja, o valor de $\mathrm{R} \$ 20.000,00$ e $\mathrm{R} \$ 40.000,00$, tais níveis de custos podem estar associados, por exemplo, à existência de incentivos fiscais, tributários ou até sobre a doação do terreno por parte da prefeitura, visto que a organização disponibilizará a sociedade novos postos de trabalho.

Como resultado da situação exposta na Figura 8, na Figura 9 é demonstrado o comportamento ao longo do tempo das seguintes variáveis, a saber: receita, custo de produção, lucro e custos fixos.

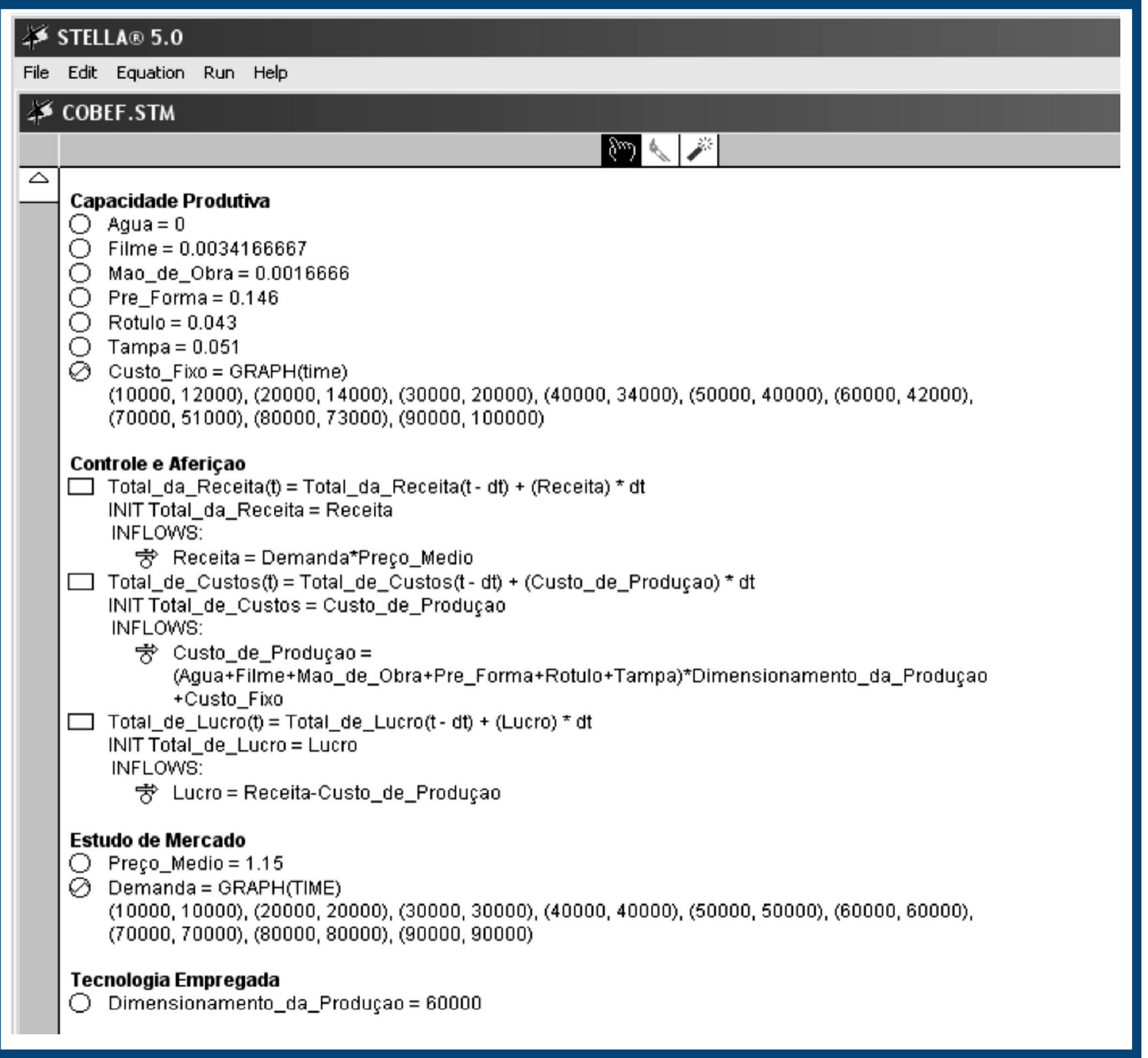

Figura 7 -Variáveis e Equações Resultantes. 
Vinicius Amorim Sobreiro

Pedro Henrique de Sousa Leão Araújo

Marcelo Seido Nagano

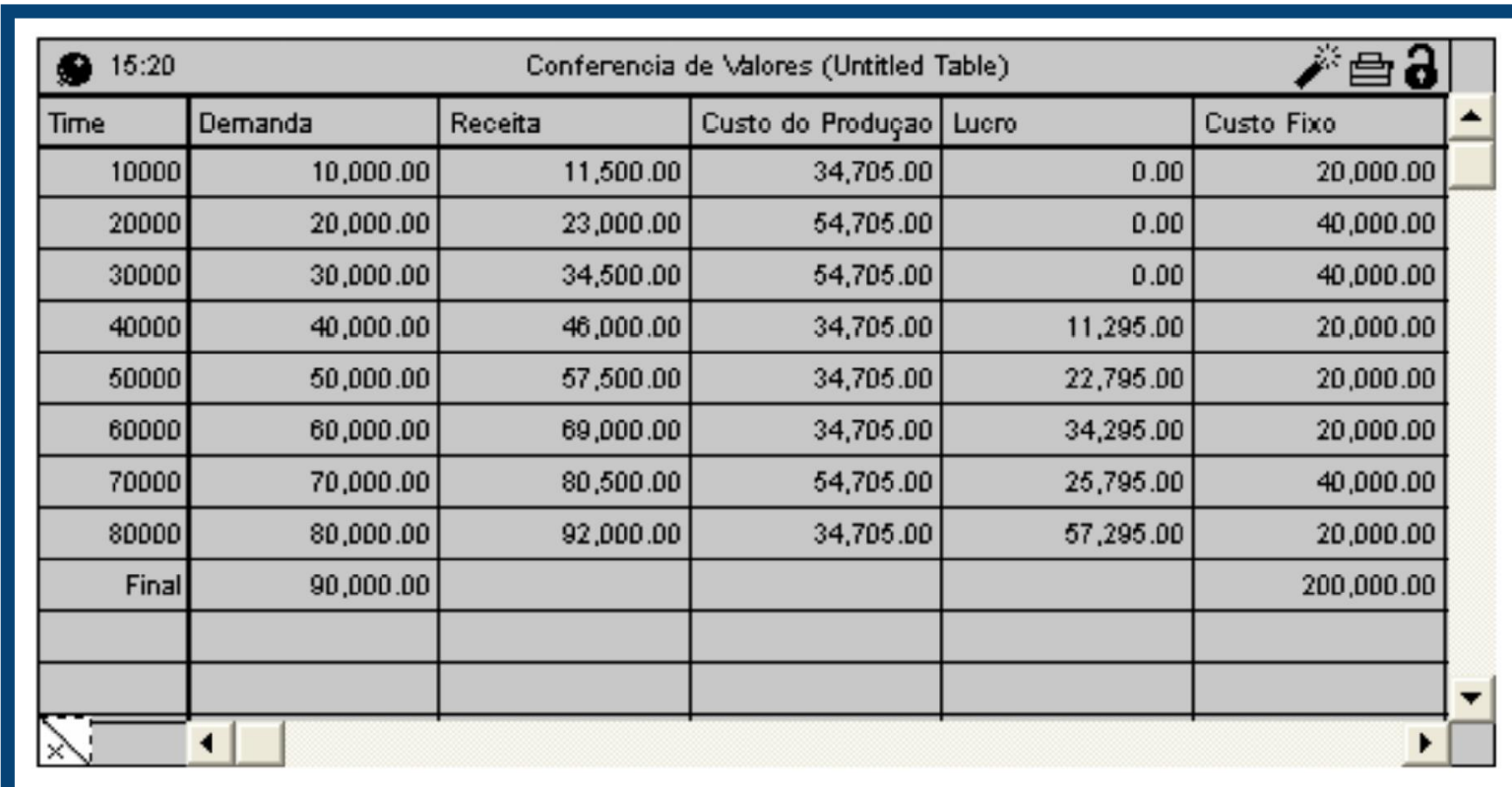

Figura 8 -Tabela de resultados para nova simulação.

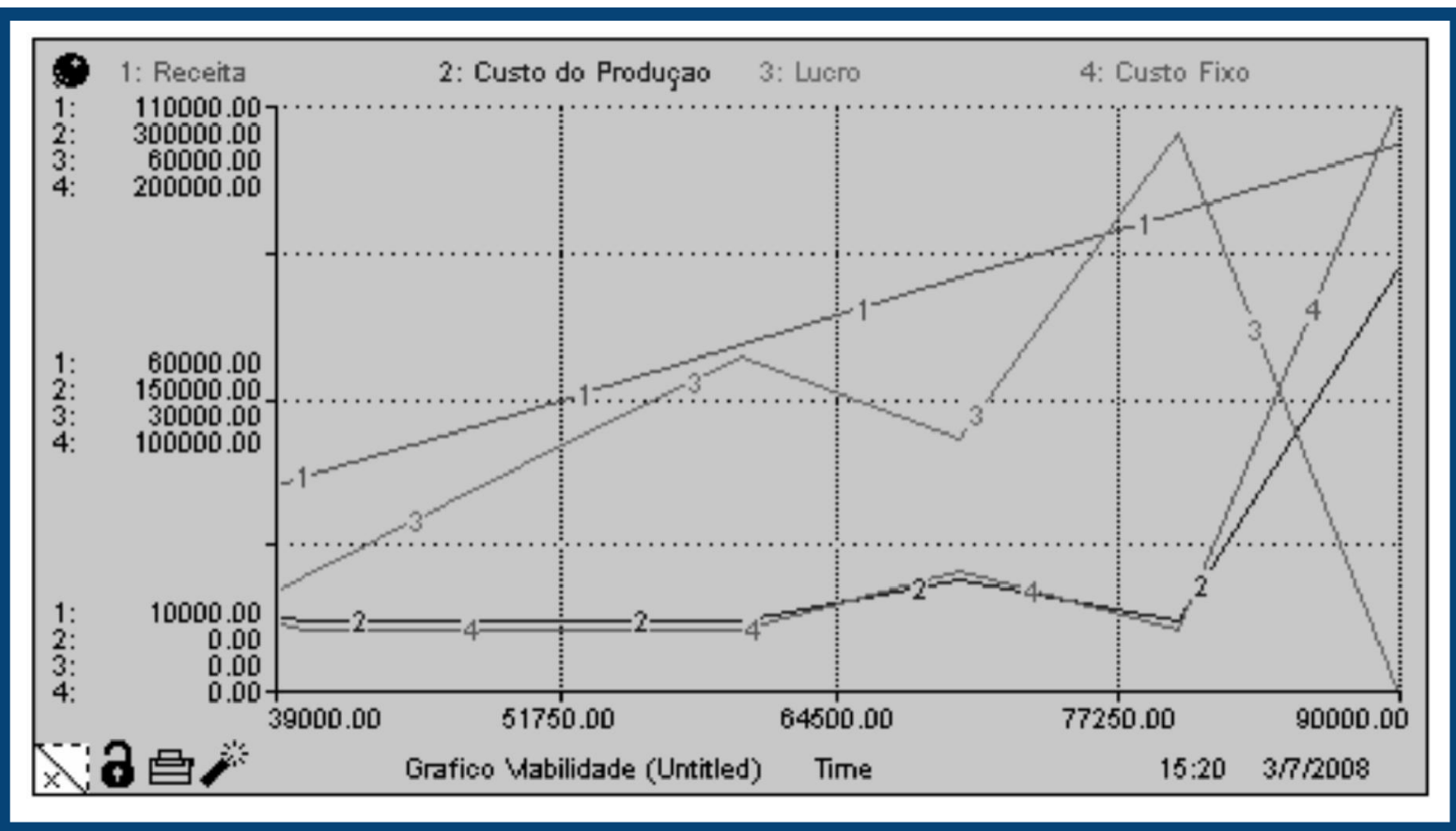

Figura 9 - Gráfico de resultados da nova simulação.

Com base na exposição dessas indicações, os resultados demonstram que:

I O empreendimento é considerado viável quanto ao seu fluxo de caixa, ou seja, levando-se em conta que a receita total estimada é maior que os custos totais. Assim, reputando a utilização dos valores demonstrados na Tabela 6 , a qual possibilita ao investidor um auxílio quantoà decisão da implementação da organização no Estado do Ceará.

। Identificação dos valores referentes aos custos fixos que, quando acrescidos dos custos variáveis, proporcionam viabilidade econômica ao empreendimento mediante as técnicas de MSD. 
I AMDS pode ser utilizada como ferramenta para a gestão e análise de custos.

Tais apontamentos possibilitam identificar que as técnicas de MSD são possíveis de aplicação para o cálculo dos custos totais, considerando-se a instalação de novas organizações ou a expansão de organizações que já estejam atuando no mercado, visto que o gerenciamento dos custos representa uma aposta de natureza excepcional sobre o futuro delas.

\section{DISCUSSÕES E CONCLUSÕES}

Planejar uma unidade de produção consiste em uma das mais complexas atividades entre as que compõem um processo de estudo de viabilidade econômico-financeira de um empreendimento. Essa dificuldade é intensificada ao existirem restrições sobre aspectos financeiros, como os custos totais de produção, tributos e margem de contribuição. Não obstante essa dificuldade, sabese que o preço é regido pelo mercado consumidor, condicionando, assim, os custos de produção a proporcionarem intervalos viáveis para a obtenção de superávits.

Nesse sentido, o processo produtivo ou sistema produtivo quando analisado de forma minuciosa constitui-se em um sistema complexo no qual se busca, mediante modelos matemáticos desenvolvidos pelos mais diversos campos das ciências da engenharia, otimizar os escassos recursos.

A crescente exposição das organizações à concorrência mundial exige dos gestores de negócios atualização e aprofundamento nas técnicas de análise pouco experimentadas pelos gestores brasileiros em geral até o início dos anos de 1990. Dentro desse cenário, surge a possibilidade apontada por diversos pesquisadores de aplicar a MSD no processo produtivo, visto as possibilidades de simular o relacionamento dinâmico e influências das principais variáveis do processo produtivo em um ambiente computacional.

Vigente a realidade de que a gestão eficiente dos custos totais de produção é um imperativo para a sobrevivência das organizações, cabe aos gestores de produção investigar o intervalo de valores de custo fixo que favorecem o sucesso do empreendimento. Com o propósito de verificar essa aplicação, realizou-se um estudo de caso, seguindo os métodos expostos na seção 3, nos alunos de graduação em Engenharia de Produção pertencentes a uma empresa júnior para identificação das con- dições de custo que propiciam à implementação de uma organização, a fim de envasar água mineral no Ceará.

Os resultados obtidos por meio desta pesquisa confirmam o grande potencial de aplicação de MSD na gerência e de seus custos, complementando parte da lacuna na área do conhecimento relacionado à Contabilidade de Custos e a Sistemas Dinâmicos, ou seja, a integração da análise de custos com a MSD, buscando identificar os custos fixos que, atribuídos aos variáveis, permitem a organização auferir lucros, criar ou manter vantagens competitivas e atingir a viabilidade econômica.

Cabe ressaltar, porém, que as limitações desta pesquisa estão centradas na diversificação e heterogeneidade dos produtos do estudo de caso, visto que o empreendimento apresentava apenas um produto, água engarrafada. Caso essas limitações não existam, é necessária a utilização de outro sistema de custeio. Nesse sentido, os autores recomendam que, caso a simulação seja realizada para uma organização com vários produtos, $\mathrm{o}$ sistema de custeio mais apropriado é o baseado em atividade $(\mathrm{ABC})$, tendo em vista as vantagens que ele apresenta quanto ao rateio dos custos fixos.

Este trabalho possibilitou aos autores proporem a realização de futuras aplicações de MSD no sistema produtivo, abordando o enfoque em controle da qualidade no processo produtivo e seus custos. Tal proposta se fundamenta na possibilidade da utilização de distribuições estatísticas na definição de algumas variáveis observadas. Mesmo não sendo consideradas na grande maioria das tentativas de representações fictícias para processos reais, há razões para se crer que, num futuro não muito distante, ter-se-á esse tipo de configuração na solução de diversos problemas.

Além disso, deve ser enfatizado que, apesar de a tendência atual ser a de obter resultados cada vez mais precisos para os fenômenos de teor econômico, é quase impossível fazer análises puramente frias e numéricas, isolando as complexas reações do homem no contexto dessas atividades. Assim, para a realização de estudos futuros se recomenda a contemplação de variáveis de teor qualitativo.

\section{REFERÊNCIAS}

ABBOTT, M. D.; STANLEY, R. S. Modeling groundwater recharge and flow in a upland fracture bedrock aquifer. System Dynamic R eview, n. 15, p. 163-184, 1999.

ADAMIDES, E. D.; VOUTSINA, M. The double-helix model of manufacturing and marketing strategies. International J ournal of Production Economics, n. 104, p. 3-18, 2006. 
Vinicius Amorim Sobreiro

Pedro Henrique de Sousa Leão Araújo

Marcelo Seido Nagano

BORNIA, A. C. A nálise gerencial de custos: aplicação em empresas modernas. Porto Alegre: Bookman, 2005. 203 p.

BRIERLEY, J.; COWTON, C. J.; DRURY, C. A comparison of product costing practices in discrete-part and assembly manufacturing and continuous production process manufacturing. International J ournal of Production Economics, n. 100, p. 314-321, 2006.

BRUNI, A. Ll.; FAMÁ, R. G estão de custos e formação de preços: com aplicações na calculadora HP 12c e Excel. 3. ed. São Paulo: Atlas, 2004. 179 p.

CLARK, J. M. Studies in the economics of overhead costs. Chicago: Univ Of Chicago, 1962. 515 p.

DEATON, M. L.; WINEBRAKE, J. J. Dynamic Modelling of Environmental Systems. J ournal of Photosynthetica, v. 38, n. 3, 2001.

DHAVALE, D. G. Activity-Based Costing In Cellular Manufacturing Systems. Industrial Engineering, n. 24, p. 44-46, 1992.

DRURY, C.; TAYLES, M. Profitability analysis in UK organizations: An exploratory study. The B ritish A ccounting R eview, v. 38, Issue 4, p. 405-425, december 2006.

DYSON, B.; CHANG, N. B. Forecasting municipal solid waste generation in a fast-growing urban region with system dynamics modeling. Waste M anagement, n. 25, p. 669-679, 2005.

FLOOD, R. L.; JACKSON, M. C. Creative Problem Solving: Total Systems Intervention. New York: John Wiley \& Sons, 1991.

FORD, A. M odeling the Environment. Washington: Island Press, 1999. 254 p.

FORRESTER, J. W. Urban dynamics. Boston: MIT Press, 1971. 35 p.

FORRESTER, J. W. Industrial dynamics. Boston: MIT Press, 1969. $20 \mathrm{p}$.

FORRESTER, J. W. Urban dynamics. Boston: MIT Press, 1961. 15 p.

GRANT, W. E.; PEDERSEN, E. K.; MARIN, S. L. Ecology and Natural Resource M anagement: Systems Analysis and Simulation. New York: Wiley, 1997.

GUO, H. C.; LIU, L.; HUANG, G. H.; FULLER, G. A.; ZOU, R.; YIN, Y.Y. A system dynamics approach for regional environmental planning and management: a study for Lake Erhai Basin. J ournal Environmental. M anagement, n. 61, p. 93-111, 2001.

JOHNSON, H. T.; KAPLAN, R. S. Relevance lost: the rise and fall of management accounting. Boston: Harvard Business School Press, 1991. 269 p.

JONES, T. C.; DUGDALE, D. The ABC bandwagon and the juggernaut of modernity. Accounting Organizations and Society, n. 27, p. 121-163, 2002.

LUCAS, M. R. Pricing decisions and the neoclassical theory of the firm. M anagement Account R esearch, n. 14, p. 201-217, 2002.

MEADOWS, D. L.; MEADOWS, D. H. Toward global equilibrium. Massachusestts: Wright-Allen Press, 1973.

MEADOWS, D. L.; MEADOWS, D. H.; RANDRES, J.; BEHRENS, W. W. The limits to growth. New York: New American Library, 1972.
NAIL, R. F.; GELANGER, S.; KLINGER, A.; PETERSON, E. An analysis of cost effectiveness of US energy policies to mitigate global warming. System Dynamic Review, n. 8, p. 111-118, 1992.

NEVES, S.; VICECONTI, P. E. V. Contabilidade de custos: um enfoque direto e objetivo. 7. ed. São Paulo: Frase, 2003. 272 p.

NOSBISCH, M. R.; WINTER, R. M. Managing Resource Leveling. Cost Engineering, v. 48, n. 7, p. 24-35, 2006.

QU, W.; BARNEY, G. O. Projecting China's grain supply and demand using a new computer simulation model. Arlington: Millennium Institute, 1998.

REIBSTEIN, D. J.; DAY G. A Dinâmica da E stratégia Competitiva. [S.1.]: Editora Campus, 1999.

RIZZO, D.M.; MOUSER, P. J.; WHITNEY, D. H.; MARK, C. D.; MAGAREY, R. D.; VOINOV, A. A. The Comparison of four dynamic systems-based software packages Translation and Sensitivity Analysis. Environmental Modelling \& Software, n. 21, p. 1491-1502, 2006.

ROSA, S. E. S.; COSENZA, J. P.; LEÃO, L. T. S. Panorama do Setor de Bebidas no Brasil. 23. ed. Rio de Janeiro: Bndes Setorial, 2006. 50 p.

SANTOS, J. J. A nál ise de custos: remodelado com ênfase para sistema de custeio marginal, relatórios e estudos de casos. 4. ed. São Paulo: Atlas, 2005. $231 \mathrm{p}$.

SAYSEL, A. K.; BARLAS, Y.; YENIGUN, O. Environmental sustainability in an agricultural development project: a system dynamics approach. J ournal Environmental M anagement, n. 64, p. 247-260, 2002.

SOUSA, G. W. L.; CARPINETTI, L. C. R. Modelagem de Dinâmica de Sistemas para Análise e Reprojeto de Empresas. In: GUERRINI, F. M. (Org.). G estão avançada de manufatura - Coleção Fábrica do Milênio. São Carlos: Cubo Multimídia, 2005. v. 2, p. 159-170.

SPEDDING, T. A.; SUN, G. Q. Application of discrete event simulation to the activity based costing of manufacturing systems. International J ournal of Production Economics, n. 58, p. 289-301, 1999.

STERMAN, J. D. Business dynamics: systems thinking and modeling for a complex world. Chicago: McGraw-Hill, 2000.

TSAI, W. H.; LAI, C. W. Outsourcing or capacity expansions: application of activity-based costing model on joint products decisions. Computers \& Operations R esearch. [S.1.]: Corrected Proof., Available online 6 March 2006. In press.

VEZJAK, M.; SAVSEK, T.; STUHLER, E. A. System dynamics of euthrophication processes in lakes. European J ournal Operation Research, n. 109, p. 442-451, 1998.

VIZAYAKUMAR, K.; MOHAPATRA, P. K. J. Environmental impact analysis of a coalfield. J ournal E nvironmental M anagement, n. 34, p. 73-93, 1991.

VIZAYAKUMAR, K.; MOHAPATRA, P. K. J. Modeling and simulation of environmental impacts of a coalfield: system dynamic approach.J ournal E nvir onmental M anagement, n. 42, p. 59-73, 1993.

WOOD, T. S.; SHELLEY, M.L. A dynamic model of bioavailability of metals in constructed wetland sediments. Ecological Engineering, n.12, p. 231-252, 1999. 
